\title{
Absence of TI-VAMP/Vamp7 Leads to Increased Anxiety in Mice
}

\author{
Lydia Danglot, ${ }^{1,2 \star}$ Kathleen Zylbersztejn, ${ }^{1,2 \star}$ Maja Petkovic, ${ }^{1,2 \star}$ Maxime Gauberti, ${ }^{3}$ Hamid Meziane, ${ }^{4}$ Roy Combe, ${ }^{4}$ \\ Marie-France Champy, ${ }^{4}$ Marie-Christine Birling, ${ }^{4}$ Guillaume Pavlovic, ${ }^{4}$ Jean-Charles Bizot, ${ }^{5}$ Fabrice Trovero, ${ }^{5}$ \\ Floriana Della Ragione, ${ }^{6,7}$ Véronique Proux-Gillardeaux, ${ }^{1,2}$ Tania Sorg, ${ }^{4}$ Denis Vivien, ${ }^{3}$ Maurizio D'Esposito, ${ }^{6,7}$ \\ and Thierry Galli ${ }^{1,2}$ \\ ${ }^{1}$ Program in Development and Neurobiology, Institut Jacques Monod, CNRS, UMR 7592, Université Paris Diderot, Sorbonne Paris Cité, F-75205 Paris, \\ France, ${ }^{2}$ Inserm ERL U950, 'Membrane Traffic in Neuronal \& Epithelial Morphogenesis,' F-75013 Paris, France, ${ }^{3}$ Inserm UMR-S U919 “Serine proteases \\ and pathophysiology of the neurovascular unit," Université Caen Basse-Normandie, GIP Cyceron, 14000 Caen, France, ${ }^{4}$ Institut Clinique de la Souris, \\ Mouse Clinical Institute, 67404 Illkirch, France, ${ }^{5}$ Key-Obs SAS, F-45100 Orléans, France, ${ }^{6}$ Institute of Genetics and Biophysics - Adriano Buzzati-Traverso, \\ 80131 Napoli, Italy, and 7Istituto di Ricovero e Cura a carattere Scientifico Neuromed, 86077 Pozzilli, Italy
}

Vesicular (v)- and target ( $\mathrm{t}$-SNARE proteins assemble in SNARE complex to mediate membrane fusion. Tetanus neurotoxin-insensitive vesicular-associated membrane protein (TI-VAMP/VAMP7), a vesicular SNARE expressed in several cell types including neurons, was previously shown to play a major role in exocytosis involved in neurite growth in cultured neurons. Here we generated a complete constitutive knock-out by deleting the exon 3 of Vamp7. Loss of TI-VAMP expression did not lead to any striking developmental or neurological defect. Knock-out mice displayed decreased brain weight and increased third ventricle volume. Axon growth appeared normal in cultured knock-out neurons. Behavioral characterization unraveled that TI-VAMP knock-out was associated with increased anxiety. Our results thus suggest compensatory mechanisms allowing the TI-VAMP knock-out mice to fulfill major developmental processes. The phenotypic traits unraveled here further indicate an unexpected role of TI-VAMP-mediated vesicular traffic in anxiety and suggest a role for TI-VAMP in higher brain functions.

\section{Introduction}

Sybl1/Vamp7, the gene encoding tetanus neurotoxin-insensitive vesicular-associated membrane protein (TI-VAMP/VAMP7), was originally identified as the first pseudoautosomal gene to be $\mathrm{X}$ and $\mathrm{Y}$ inactivated (D'Esposito et al., 1996). TI-VAMP is a vesicular soluble $N$-ethylmaleimide-sensitive fusion protein attachment protein receptor (SNARE) (Galli et al., 1998). The pairing of vesicular ( $\mathrm{v}$ )- and target $(\mathrm{t})$-SNAREs located on two opposing membranes leads to subsequent membrane fusion (Weber et al., 1998). TI-VAMP is resistant to clostridial neurotoxins unlike

Received Aug. 30, 2011; revised Nov. 28, 2011; accepted Dec. 15, 2011.

Author contributions: L.D., D.V., and T.G. designed research; L.D., K.Z., M.P., M.G., H.M., R.C., M.-F.C., M.-C.B., G.P., J.-C.B., and F.T. performed research; L.D., K.Z., M.P., M.G., H.M., R.C., M.-F.C., M.-C.B., G.P., J.-C.B., F.T., F.D.R., V.P.-G., T.S., D.V., M.D., and T.G. analyzed data; L.D., K.Z., M.P., M.G., V.P.-G., T.S., D.V., M.D., and T.G. wrote the paper.

Work in our group was funded in part by grants from Inserm, the Association Française contre les Myopathies, the Association pour la Recherche sur le Cancer, the Mairie de Paris Medical Research and Health Program, the Fondation pour la Recherche Médicale (FRM), and the Ecole des Neurosciences de Paris (ENP) (to T.G.). K.Z. was supported by a Bourse de doctorat pour ingénieurs fellowship from the CNRS and a doctoral fellowship from the FRM. M.P. was supported by the ENP Ph.D. program. The Institut Clinique de la Souris - ICS (Mouse Clinical Institute) was funded by Inserm. We thank Nicolas Cogrel for his excellent technical assistance in the elevated-plus-maze and cookie tests. We are grateful to F. Causeret, Y. Arai, and A. Pierani (Institut Jacques Monod, Paris) for helpful discussion. We are respectively grateful to A. Peden (MRC, Cambridge, UK) and B. Gasnier (Université Paris Descartes) for the kind gift of anti-VAMP7 and anti-VIAAT antibody.

*L.D., K.Z., and M.P. contributed equally.

Correspondence should be addressed to Thierry Galli, Institut Jacques Monod, Bat. Buffon, 15 rue Hélène Brion, 75205 Paris Cedex 13, FRANCE. E-mail: thierry.galli@inserm.fr.

DOI:10.1523/JNEUROSCI.4436-11.2012

Copyright $\odot 2012$ the authors $\quad 0270-6474 / 12 / 321962-07 \$ 15.00 / 0$
VAMPs $1 / 2 / 3$, defining clostridial neurotoxin-resistant pathways. TI-VAMP contains an N-terminal extension of $\sim 100$ aa called Longin domain which has two functions: it binds to the $\delta$ subunit of the molecular coat AP-3 to target TI-VAMP to synaptic vesicles (Scheuber et al., 2006), and it inhibits the formation of SNARE complexes (Martinez-Arca et al., 2003). TI-VAMP interacts with plasma membrane and endosomal t-SNAREs, and is necessary for lysosomal and granule secretion in several cell types (for review, see Chaineau et al., 2009). In neurons, TI-VAMP was shown to play important role for axonogenesis (Gupton and Gertler, 2010), neurite growth in PC12 cells (Martinez-Arca et al., 2000; Racchetti et al., 2010), and axonal and dendritic growth in cultured neurons (Martinez-Arca et al., 2001; Alberts et al., 2003). Here, we set out to analyze the function of TI-VAMP in mouse behavior following the generation of a knock-out mutant.

\section{Materials and Methods}

Mouse line creation and reverse transcription-PCR. The VAMP7 knockout mouse line was established at the Institut Clinique de la Souris (ICS; Illkirch, France; http://www.ics-mci.fr/). The targeting vector was constructed by successive cloning of PCR products and contained $4.2 \mathrm{~kb} \mathrm{5}$, $0.6 \mathrm{~kb}$ floxed and $3.6 \mathrm{~kb} \mathrm{3'}$ homology arms and a Neomycin selection cassette. Two loxP sequences, delimiting the floxed arm, were located directly in intronic sequences bordering exon 3 .

The linearized construct was electroporated in 129S2/SvPas mouse embryonic stem (ES) cells. After selection by PCR and Southern blot (data not shown), a positive ES clone was injected into C57BL/6J blastocysts, and male chimaeras derived gave germline transmission. The resulting line was 
checked by PCR and bred with a CMV-Cre deleter mouse to result in deletion of exon 3. Progeny mice were backcrossed on C57BL/6 background. The mice were subsequently genotyped using the forward primer $\left(5^{\prime}\right.$ CGGTAACTGATGGTTTTACAATCTG-3') and the reverse primer $\left(5^{\prime}-\right.$ GTTGATAAAGGTACAGAGAG-3'), WT amplified sequence being $730 \mathrm{bp}$ while the knock-out amplification was $250 \mathrm{bp}$. For reverse transcription (RT)-PCR, forward primer was 5' -CCCGCCAGACGGTACTCGGT-3' and reverse primer was 5'-AGTTTGGTGGTTTCCACCTTGGCA-3'.

Neuronal cell cultures. Hippocampal cultures from 18-d-old embryo were prepared, fixed, and stained as described previously (Danglot et al., 2003). Cells were dissociated and plated on poly-L-lysine $(1 \mathrm{mg} / \mathrm{ml}$, Sigma)-coated glass coverslips.

Immunocytochemistry and Western blot. Immunocytochemistry was performed as previously described (Scheuber et al., 2006). Images of tissue section were acquired on a Leica SP5 confocal microscope by sequential scanning of the emission lines and manually adjusted to obtain the entire brain surface. Fluorescence images were acquired on a Leica DMRE microscope using FITC-, Cy3-, and DAPI-specific sets of filters and a highresolution camera (Coolsnap HQ) driven by MetaMorph. Axons and dendrites' lengths were measured using MetaMorph. Statistical significance was evaluated using GraphPad Prism. The level of significance (MannWhitney) is indicated by one $(p<0.05)$, two $(p<0.01)$, or three $(p<$ $0.001)$ symbols. Tissues were quickly dissected and homogenized in TSE (Tris/sodium/EDTA) buffer and processed for Western blotting as described previously (Danglot et al., 2010). Antibodies against TI-VAMP, Syb2, VAMP4, SNAP-25, SNAP-23, Syntaxin 1, Synaptophysin, Varp, L1-CAM, AP-3, VIAAT were previously described (Alberts et al., 2003; Martinez-Arca et al., 2003; Burgo et al., 2009; Danglot et al., 2010). All other antibodies against tubulin (Hybridoma Bank), GFP and Tau (Roche Diagnostics), HRB (Santa Cruz Biotechnology), MAP2 (Abcam), GAP-43 (Millipore), Syntaxin 3 (Sigma), Golgin (BD Biosciences) were commercially available.

Animal production and phenotyping. The WT and KO littermate male cohort was established at the Mouse Clinical Institute animal facility. They were 10 weeks old at the beginning of testing and had a mixed genetic background 129/Sv-C57BL/6. They were housed in M.I.C.E. cages and maintained in a room with controlled temperature $\left(21-22^{\circ} \mathrm{C}\right)$ under a $12 \mathrm{~h}$ light/dark cycle (light on at 7:00 A.M.), with food and water available ad libitum, with a standard chow diet (Safe D04) during the whole protocol. All experiments were performed in accordance with the European Communities Council Directive of 24 November 1986. Modified SHIRPA, rotarod test, grip test, tail flick, hot plate, open field test, tail suspension, auditory startle reflex reactivity and prepulse inhibition, Y-maze spontaneous alternation task (Meziane et al., 2007; Mandillo et al., 2008), string test (Andrieu et al., 2006), shock threshold test, and Pavlovian fear conditioning (Lamprianou et al., 2006) were previously described. Noninvasive blood pressure was performed in conscious animals by tail cuff sphygmomanometry as described previously (Koutnikova et al., 2009). Blood was collected after $4 \mathrm{~h}$ fasting by retro-orbital puncture upon isoflurane anesthesia, and blood parameters analyzed as described previously (Champy et al., 2004). Data were analyzed using unpaired Student's $t$ test, one factor or repeated measures ANOVA with one between factor (genotype) and one within factor (time). Qualitative parameters (e.g., some clinical observations) were analyzed using $\chi^{2}$ test. The level of significance was set at $p<0.05$. In vivo magnetic resonance imaging was performed according to the method of Gakuba et al. (2011).

Cookie test and elevated-plus-maze test. These tests were conducted at KeyObs on 17-week-old males produced at ICS, housed in groups of 2-5. Olfactory function was measured in the cookie test as described previously (Dawson et al., 2005). Anxiety-related behavior was measured in the elevated-plus-maze test as described previously (Belzung and Griebel, 2001).

\section{Results}

Vamp7 gene targeting strategy and expression analysis in KO mice

We generated a Vamp7 knock-out mouse line (called TI-VAMP $\mathrm{KO}$ or $\mathrm{KO}$ ) by invalidating the X-linked Vamp7 gene in a CreloxP system to avoid Vamp7 nullisomy at the level of the male ES cells. A Vamp7 floxed allele (Vamp $7^{\text {flox/flox }}$ ) was created by insert- ing loxP sequences at the borders of exon 3 (Fig. 1A) and a Vamp7 $\mathrm{KO}$ allele lacking exon 3 by crossing homozygous $V a m p 7^{\text {flox/flox }}$ mice with a $C M V$-Cre deleter line expressing the Cre recombinase early during embryonic development and active in most cells and tissues (Fig. 1A). The CMV-Cre::Vamp $7^{\text {flox/flox }}$ strain was crossed with C57BL/6 WT animals to eliminate the CMV-Cre transgene. The resulting mice, referred to as TI-VAMP KO, develop normally up to adulthood.

We first checked mRNA expression transcripts using primers selected in the first and the last exon. We obtained one single band corresponding to the full mRNA sequence in WT and the expected recombined band in $\mathrm{KO}$. In $\mathrm{KO}$, however, we also noted minor RT-PCR fragments which by sequencing were found to lack exon 3. These fragments included a potential open reading frame with an ATG in exon 5 (Vacca et al., 2011; 3' fragment). We also found the previously identified isoform c (TIVc; Martinez-Arca et al., 2003). Thus, we further checked whether the mRNAs found in KO could encode a C-terminal fragment of TI-VAMP. To this end, we produced artificial proteins (full-length TI-VAMP, 3' fragment and TIVc) in vitro in rabbit reticulocytes. Translated TI-VAMP, $3^{\prime}$ fragment and TIVc were immunoprecipitated with a pAb (TG18), then recognized by anti-TI-VAMP mAb158.2 (Fig. $1 B$ ). Furthermore, expression of GFP-tagged TIVc in Cos7 cells allowed us to confirm that both our antibodies recognize TIVc (Fig. 1C). Using these tools, we found that TI-VAMP KO mice consistently displayed no expression of full-length TI-VAMP, $3^{\prime}$ or TIVc encoded proteins (Fig. 1D). To test whether even a low amount of $3^{\prime}$ or TIVc could be expressed, immunoprecipitations by TG18 from both cortex and liver tissues were performed. Full-length TI-VAMP was immunoprecipitated in WT tissues, whereas neither $3^{\prime}$ nor TIV c were detected in WT or KO cortex or liver (Fig. 1E). These data demonstrate that TI-VAMP KO is a null allele and that the mRNA identified do not lead to expression of truncated forms of the protein. We further found that other neuronal $v$ - and t-SNAREs as well as TI-VAMP partners, Varp and HRB (Chaineau et al., 2008; Burgo et al., 2009), were expressed at similar levels in WT and $\mathrm{KO}$ (Fig. $1 F$ ).

\section{Decreased body temperature and increased blood glycerol in TI-VAMP KO mice}

The TI-VAMP KO mice were viable and fertile. We had on average $8.25 \pm 0.66$ pups per litter over 15 litters (124 animals) obtained by crossing heterozygous females with $\mathrm{KO}$ males, with a $50: 50$ male/female ratio ( $43.2 \pm 4.8 \%$ of females vs $56.8 \pm 4.8 \%$ of males; one sample $t$ test $p=0.1798$ ). However, we detected a significant decrease in proportion of heterozygous females $(17+$ $3.7 \%$ compared with the expected $25 \%$, one sample $t$ test $p=$ $0.0469)$. We then systematically examined male mice of the same age. $\mathrm{KO}$ males showed no difference in body weight or length, had normal physical appearance including fur pigmentation, but showed significantly lower body temperature compared with WT $(p<0.05)$ (Table 1). Because of TI-VAMP's function in cardiomyocytes (Schwenk et al., 2010), TI-VAMP knock-out was analyzed to detect cardiac and/or blood pressure disorder. TI-VAMP KO male mice showed no defect in noninvasive blood pressure measurements in conscious animals or echocardiography in anesthetized mice. We also evaluated several parameters in plasma samples (Table 1). None of these biological parameters showed significant differences between $\mathrm{KO}$ and WT except glycerol levels which were slightly higher in $\mathrm{KO}$, however still in physiological range. 
A
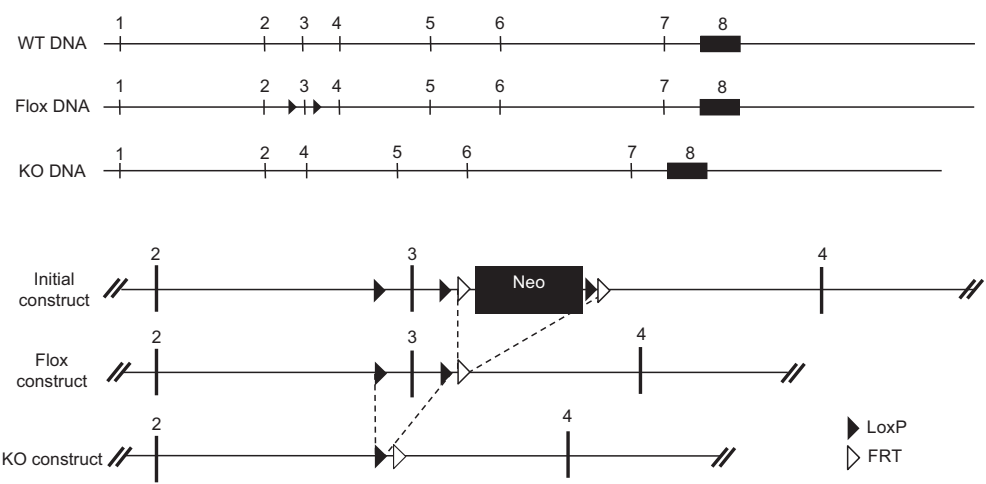

B

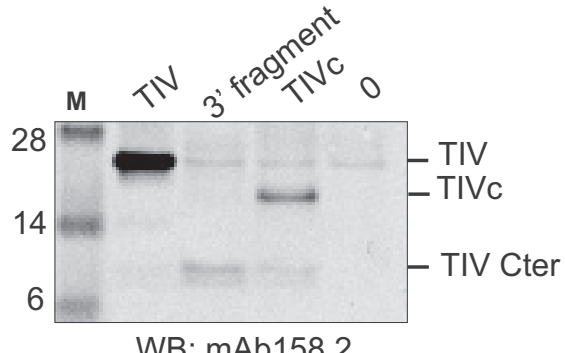

D

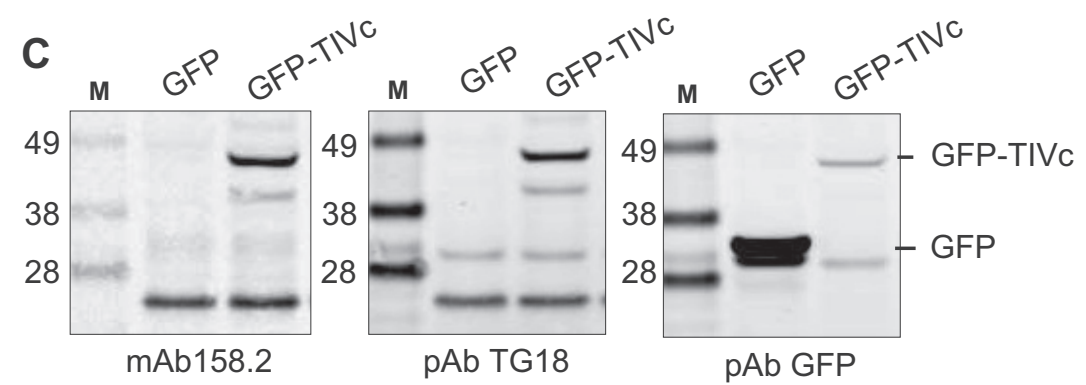

м WT KO м WT KO м WT KO м WT KO м WT KO м WT KO м WT KO м WT KO м WT KO

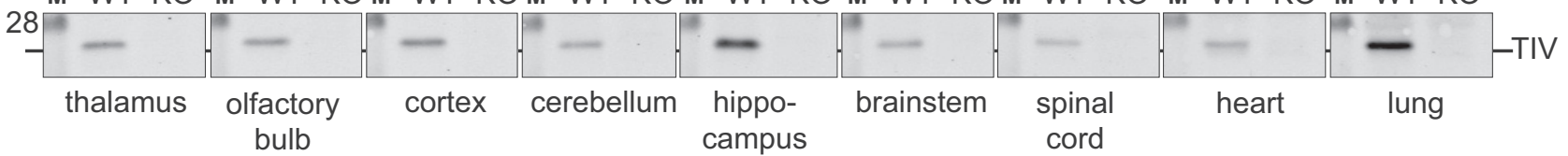

E Cortex WT

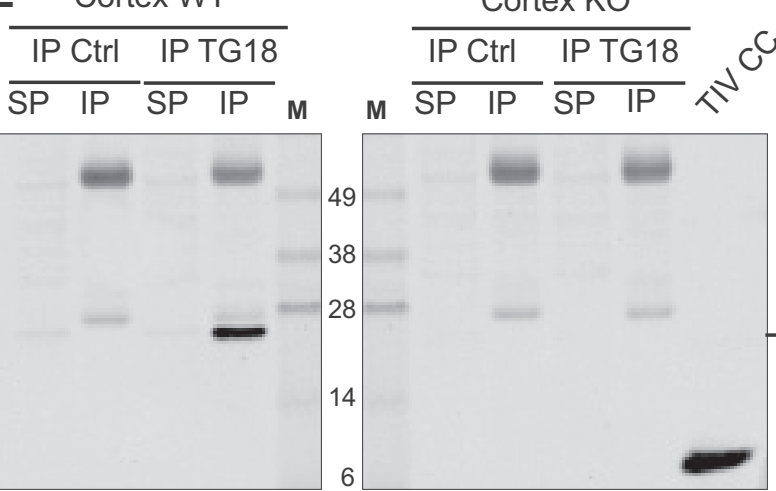

WB: mAb158.2
Cortex KO

cord

Liver KO

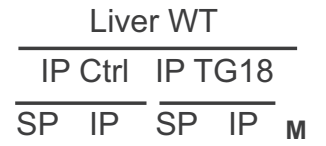

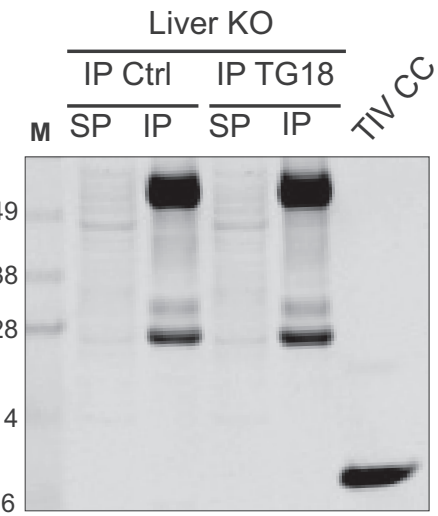

WB: mAb158.2
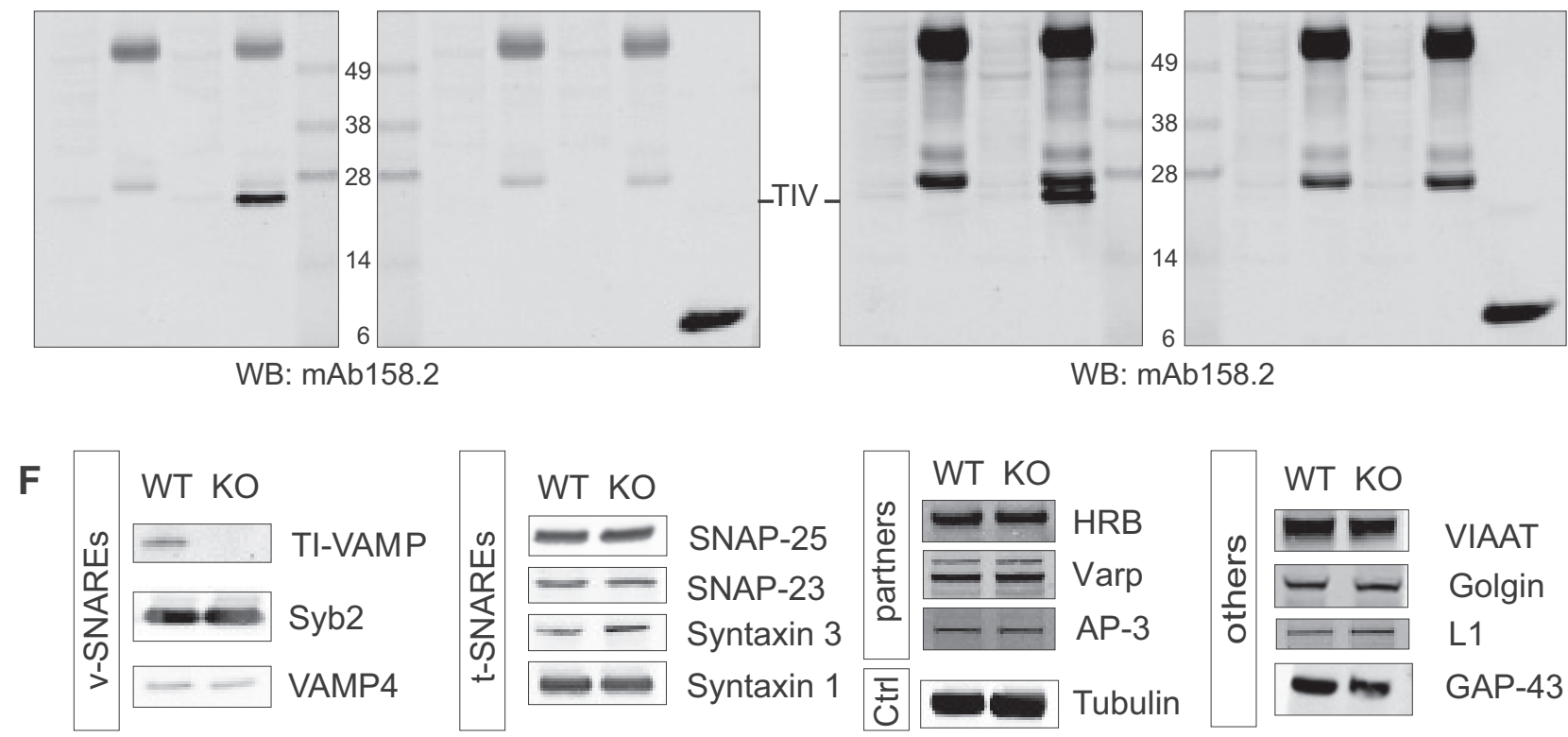

VIAAT

Golgin

L1

GAP-43

Figure 1. CMV-Cre::Vamp $7^{\text {floxfflox }}$ mice are full Vamp7 knock-out mice. $A$, Strategy for the CMV-Cre::Vamp7-flox/flox construct. B, TI-VAMP, $3^{\prime}$ fragment (TIV-Cter) and TI-VAMPc (TIVc) CDNAs were used to produced proteins in rabbit reticulocytes $(0=$ no plasmid), which were immunoprecipitated with pAb TG18 and revealed with 158.2 mAb. C, GFP-TI-VAMPc expressed in Cos cells is recognized by mAb158.2, pAbTG18 and anti-GFP pAbs. D, Extracts of indicated tissues from WT and K0 were Western blotted with mAb158.2. E, Immunoprecipitation with TG18 and rabbit IgG (control) of cortex and liver extracts from WT and K0 littermates were revealed with mAb158.2. As control, $25 \mathrm{ng}$ of TI-VAMP coiled-coil (aa 119-189, CC) was loaded. SP, Supernatant; IP, immunoprecipitation. $\boldsymbol{F}$, Extracts of WT and KO cortex $(40 \mu \mathrm{g})$ were Western blotted for the indicated proteins. 
Table 1. Lower body temperature and brain weight and increased glycerol in TI-VAMP KO

\begin{tabular}{|c|c|c|}
\hline & WT & KO \\
\hline & Mean \pm SEM & Mean \pm SEM \\
\hline \multicolumn{3}{|l|}{ General Health } \\
\hline Body weight (g) & $26.64 \pm 0.62$ & $26.30 \pm 0.17$ \\
\hline Brain weight (g) & $0.47 \pm 0.006$ & $0.43 \pm 0.015^{*}$ \\
\hline Body length (cm) & $9.75 \pm 0.08$ & $9.55 \pm 0.11$ \\
\hline Body temperature $\left({ }^{\circ} \mathrm{C}\right)$ & $36.98 \pm 0.26$ & $36.09 \pm 0.25^{*}$ \\
\hline \multicolumn{3}{|l|}{ Blood chemistry } \\
\hline Sodium & $149.91 \pm 0.34$ & $150.31 \pm 0.55$ \\
\hline Potassium & $4.66 \pm 0.08$ & $4.56 \pm 0.15$ \\
\hline Chloride & $112.73 \pm 0.41$ & $113.31 \pm 0.29$ \\
\hline Calcium & $2.41 \pm 0.01$ & $2.45 \pm 0.02$ \\
\hline Phosphorus & $2.33 \pm 0.16$ & $2.23 \pm 0.13$ \\
\hline Total proteins & $51.55 \pm 0.69$ & $51.77 \pm 0.46$ \\
\hline Urea & $7.11 \pm 0.21$ & $6.86 \pm 0.22$ \\
\hline Creatinine & $7.71 \pm 0.30$ & $7.57 \pm 0.18$ \\
\hline Albumin & $27.82 \pm 0.48$ & $27.92 \pm 0.31$ \\
\hline Total bilirubin & $2.23 \pm 0.18$ & $2.05 \pm 0.06$ \\
\hline Lactate dehydrogenase & $387.90 \pm 26.84$ & $344.38 \pm 26.99$ \\
\hline Aspartate aminotransferase & $69.40 \pm 4.95$ & $63.85 \pm 6.06$ \\
\hline Alanine aminotransferase & $40.00 \pm 1.58$ & $38.85 \pm 3.27$ \\
\hline Alkaline phosphatase & $83.91 \pm 1.39$ & $85.38 \pm 1.46$ \\
\hline$\alpha$-Amylase & $630.36 \pm 22.01$ & $666.77 \pm 20.72$ \\
\hline Glucose & $16.85 \pm 0.98$ & $18.98 \pm 0.67$ \\
\hline Total cholesterol & $2.94 \pm 0.12$ & $2.80 \pm 0.06$ \\
\hline HDL cholesterol & $2.04 \pm 0.09$ & $1.93 \pm 0.05$ \\
\hline LDL cholesterol & $0.49 \pm 0.02$ & $0.47 \pm 0.01$ \\
\hline Triglycerides & $1.17 \pm 0.07$ & $1.14 \pm 0.11$ \\
\hline Free fatty acids & $1.25 \pm 0.03$ & $1.41 \pm 0.08$ \\
\hline Glycerol & $337.63 \pm 8.46$ & $386.92 \pm 19.71^{*}$ \\
\hline Insulin & $0.66 \pm 0.23$ & $0.46 \pm 0.11$ \\
\hline Leptin & $2.19 \pm 0.43$ & $1.80 \pm 0.35$ \\
\hline Adiponectin & $10.26 \pm 0.79$ & $12.60 \pm 1.09$ \\
\hline \multicolumn{3}{|l|}{ Behavioral tests } \\
\hline \multicolumn{3}{|l|}{ Open field } \\
\hline Distance traveled (m) & $106.89 \pm 3.99$ & $116.11 \pm 4.10$ \\
\hline Number of rears & $261.55 \pm 25.17$ & $293.08 \pm 39.43$ \\
\hline$\%$ of time in the center & $12.55 \pm 1.35$ & $14.66 \pm 1.85$ \\
\hline Number of entries & $109.45 \pm 8.91$ & $119.23 \pm 8.66$ \\
\hline Rotarod- 4 to $40 \mathrm{rpm}$ in $5 \mathrm{~min}(\mathrm{~s})$ & $132.45 \pm 10.75$ & $113.62 \pm 10.54$ \\
\hline Grip strength ratio (grip mean/weight) & $7.82 \pm 0.32$ & $8.39 \pm 0.20$ \\
\hline String test (s) & $2.32 \pm 0.27$ & $3.16 \pm 0.36$ \\
\hline Tail flick (s) & $4.24 \pm 0.14$ & $4.19 \pm 0.18$ \\
\hline \multicolumn{3}{|l|}{ Hot plate } \\
\hline First reaction (s) & $9.35 \pm 0.93$ & $9.44 \pm 0.72$ \\
\hline Jump (s) & $90.13 \pm 6.63$ & $97.64 \pm 5.79$ \\
\hline \multicolumn{3}{|l|}{ Shock threshold (mA) } \\
\hline Flinch & $0.08 \pm 0.00$ & $0.08 \pm 0.00$ \\
\hline Vocalization & $0.18 \pm 0.02$ & $0.18 \pm 0.02$ \\
\hline \multicolumn{3}{|l|}{ Y-maze alternation } \\
\hline \% Spontaneous alternation & $60.40 \pm 3.49$ & $64.91 \pm 2.76$ \\
\hline$\%$ Alternance arm return & $34.82 \pm 3.75$ & $33.52 \pm 2.93$ \\
\hline \%Same arm return & $2.29 \pm 0.68$ & $0.92 \pm 0.49$ \\
\hline \multicolumn{3}{|l|}{ Tail suspension test } \\
\hline Duration of immobility (s) & $144.80 \pm 23.02$ & $154.15 \pm 20.93$ \\
\hline Latency to first immobility (s) & $70.57 \pm 18.03$ & $46.80 \pm 5.89$ \\
\hline
\end{tabular}

General health, blood chemistry analyses, and behavioral tests were performed on WT and KO littermates (WT, $n=$ $11 ; \mathrm{K} 0, n=13 ;{ }^{*} p<0.05$ by Student's $t$ test).

\section{Effect of lack of TI-VAMP on neurite growth and brain development}

Intriguingly, we found a significant decrease of $8.5 \%$ in brain weight in TI-VAMP KO mice (Table 1). No significant difference was however observed in the gross architecture of the brain as revealed by DAPI staining (Fig. $2 A$ ). We did not find any significant difference neither in the number of cells stained by BrdU, nor in staining for Doublecortin and Caspase 3 in E15.5 dorso- lateral cortex. This suggests normal cell proliferation and death in TI-VAMP KO at E15.5 (our unpublished observations). Staining of the presynaptic marker Synaptophysin in the hippocampus showed the same distribution between KO and WT whereas TIVAMP staining was totally lost in KO (Fig. $2 B$ ). Expression of several neuronal markers (L1-CAM, VIAAT, GAP-43) using Western blot showed no difference (Fig. $1 F$ ). MRI showed increased volume of the third ventricle in TI-VAMP KO mice (Fig. $2 C, D$ ). In previous studies, TI-VAMP was shown to mediate axonal and dendritic growth in cultured neurons (Martinez-Arca et al., 2001; Alberts et al., 2003). Therefore, we measured axonal length in cortical explants from WT and $\mathrm{KO}$ grown in plasma matrix but found no difference (Fig. 2E). Accordingly, hippocampal neurons grown for $2 \mathrm{DIV}$, in the presence or absence of feeding astrocytes, showed no significant defect in axonal or dendritic growth (Fig. $2 \mathrm{~F}$ ). Altogether, these findings indicate that no major developmental defect was observed in differentiating neurons in absence of TI-VAMP.

\section{Increased anxiety in TI-VAMP KO mice}

TI-VAMP was proposed to mediate the release of neurotransmitters at a subset of synapses (Scheuber et al., 2006). This led us to characterize behavioral alterations in TI-VAMP KO male mice as possible consequences of presynaptic defects. For this purpose, KO mice and their WT littermates were analyzed in a behavioral test battery designed to investigate a wide range of nervous system functions including sensory-motor abilities, emotional behavior, sensory-motor gating, olfaction, learning and memory, and anxiety. When tested for specific motor abilities, TI-VAMP KO males performed correctly in the grip, string and rotarod tests compared with WT (Table 1). In the open-field test, overall locomotor activity and rears were comparable between WT and KO (Table 1). Exploration of the central part of the open field arena was also comparable between genotypes (Table 1). TI-VAMP KO mice performed like WT in the hidden cookie test which is devoted to measure olfaction (Fig. 3A). Gross neurological evaluation revealed that mutant mice were less active than WTs after immediate transfer in SHIRPA's arena $\left(t_{22}=2.30, p<0.05\right)$ perhaps suggesting increased anxiety (see below). Sensory and vestibular functions were not affected. Mutant mice had also normal pain sensitivity in the tail flick and hot plate tests and normal foot-shock sensitivity (Table 1). Loss of TI-VAMP was further evaluated using auditory startle and prepulse inhibition (PPI) of startle reflex. The amplitude of the startle response to high sudden acoustic stimulus (110 dB) was slightly higher in TI-VAMP $\mathrm{KO}$ than in WT, but the difference between genotypes was not significant (Fig. 3B). When the startling pulse was preceded by prepulses with lower intensities, the level of PPI tended to be reduced in TI-VAMP KO mice (Fig. $3 B$ ). In the Y-maze paradigm, no difference in the ratio of spontaneous alternation was noted between WT and TI-VAMP KO mice (Table 1), thus indicating that working memory performance was not affected in KO. The number of visited arms was also comparable between the two genotypes (Table 1). We then tested the role of TI-VAMP in emotional behavior. In the tail suspension test, the latency to the first immobilization was slightly but not significantly reduced in TI-VAMP KO mice perhaps suggesting a tendency to despair (Table 1). WT and KO behaved similarly in fear conditioning (Fig. 3C). Finally, we analyzed KO and their WT littermates for the evaluation of anxiety. TI-VAMP KO mice performed as control in the Four Plates test, an animal model of anxiety based on conditioned response used to evaluate the anxiolytic-like effect of molecules (Masse et al., 2008). In the elevated-plus-maze test, the 

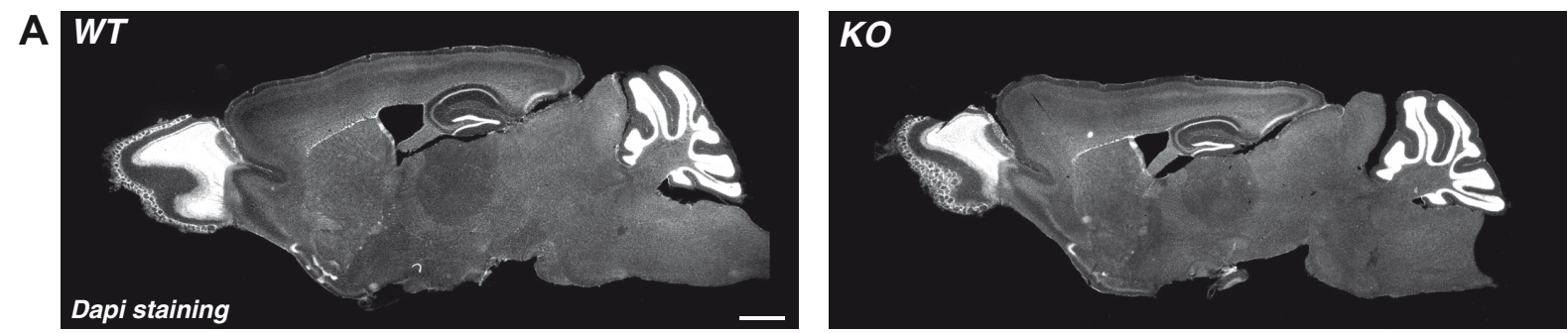

B

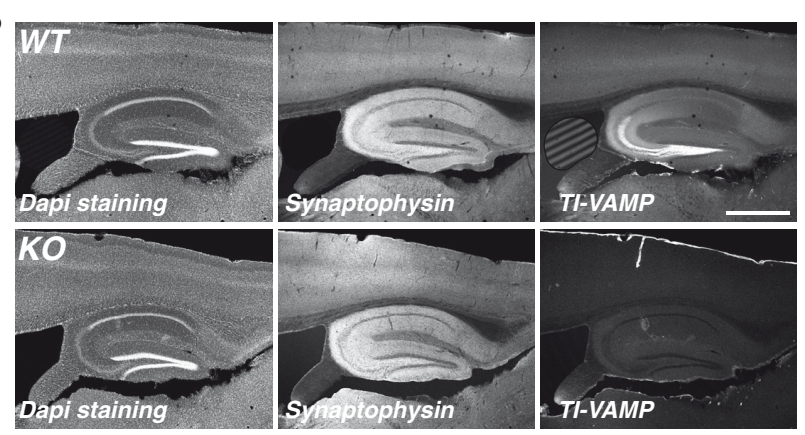

D
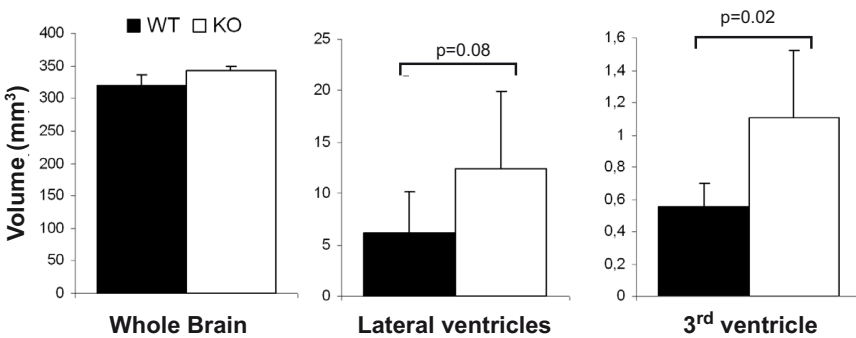

C

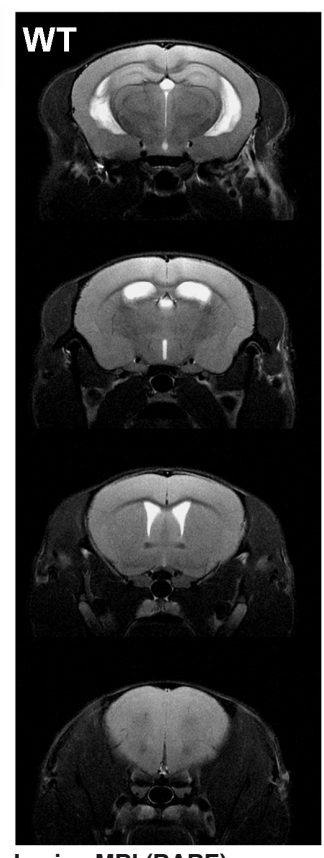

In vivo MRI (RARE).
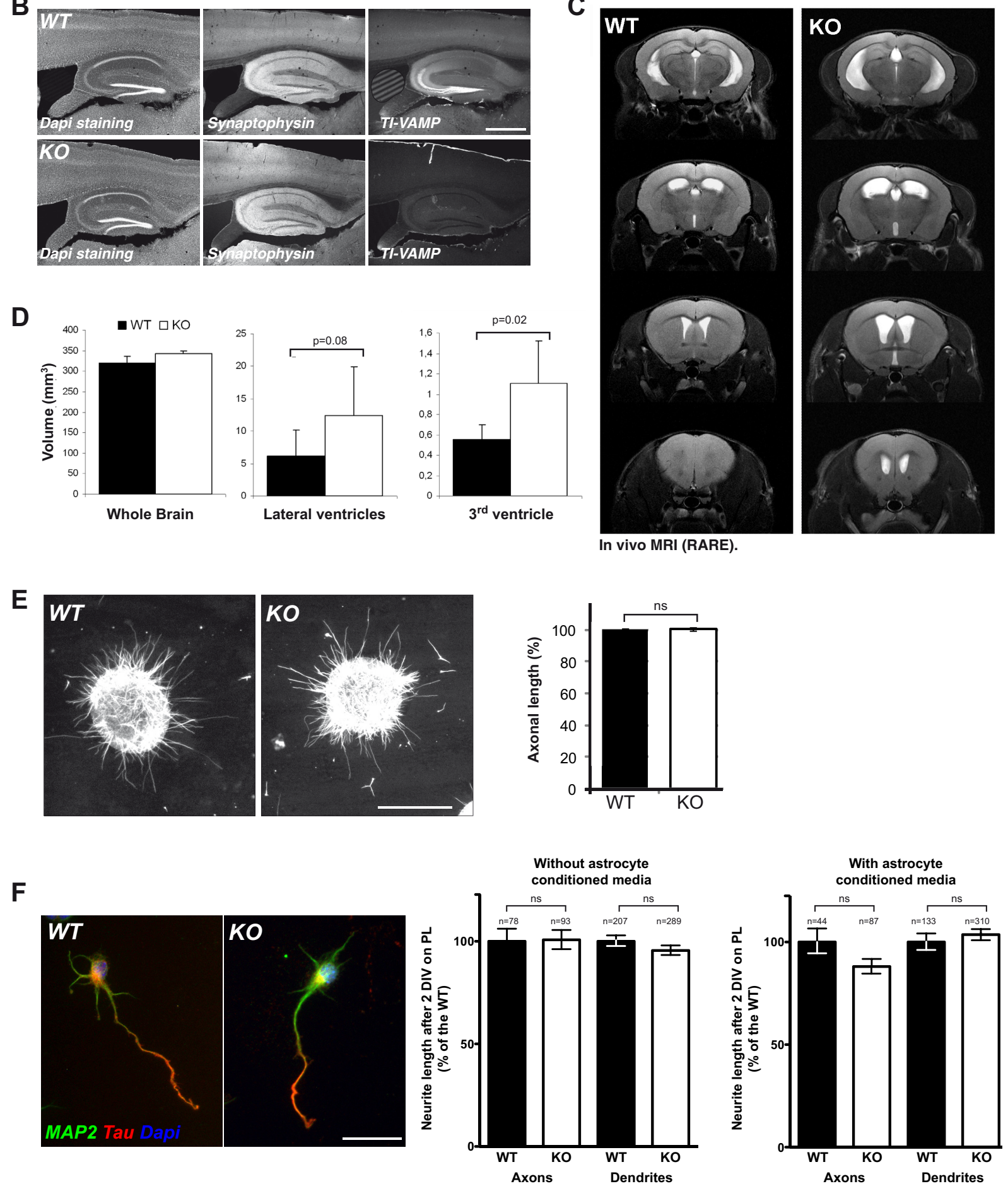

Figure 2. Gross anatomy of the brain and neurite length in TI-VAMP KO. A, Sagittal sections of WT and KO animals stained with DAPI. Fifteen confocal pictures were manually assembled to generate an image of full brain (scale bar: $500 \mu \mathrm{m}$ ). B, Sagittal sections of WT and KO hippocampus stained with DAPI, Synaptophysin and TI-VAMP. Hippocampal (Figure legend continues.) 
A

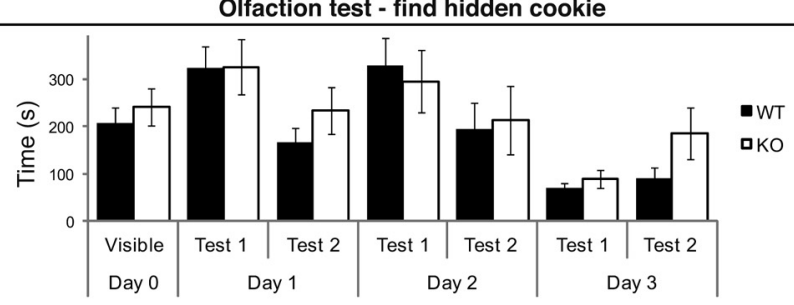

B

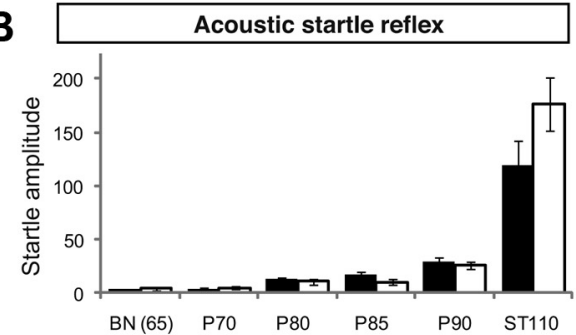

C

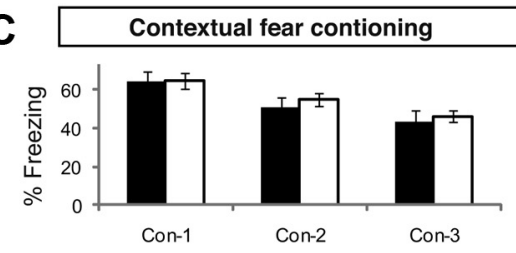

D

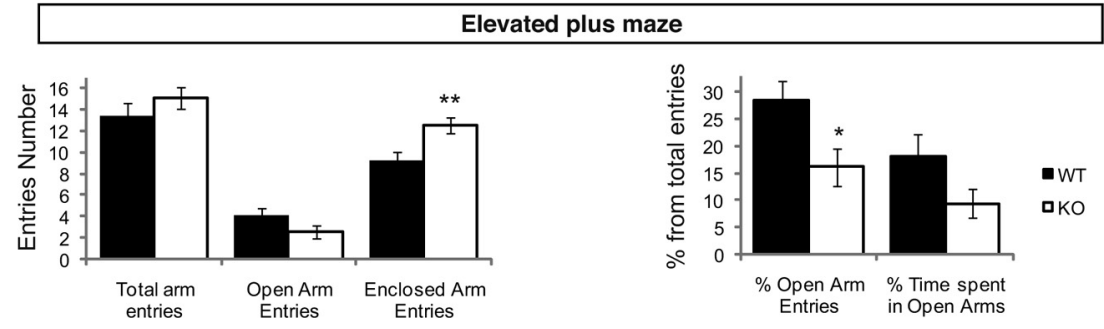

Figure 3. TI-VAMP KO show increased anxiety. $\boldsymbol{A}-\boldsymbol{D}, \mathrm{KO}$ and WT littermates were analyzed with olfaction cookie test $(\boldsymbol{A})$, acoustic startle test $(\boldsymbol{B})$, contextual and cued fear conditioning tests $(\boldsymbol{C})$, and elevated plus maze (anxiety, $\boldsymbol{D})(\mathrm{K} 0, n=11 ; \mathrm{WT}, n=$ $13 ;{ }^{* *} p<0.005$, Student's $t$ test).

percentage of entries in open arms and the time spent in open arms which are elevated from the floor are used as index of anxiety. The number of total entries was similar in both genotypes indicating a similar locomotion activity in the maze. However, there was a significant increase in enclosed arms entries and decrease in the percentage of open arm entries in TI-VAMP KO indicating a higher unconditioned basal anxiety level in the KO (Fig. 3D).

$\leftarrow$

(Figure legend continued.) region which harbors the highest expression level of TI-VAMP, is totally deprived of TI-VAMP signal in the K0, whereas the synaptic marker Synaptophysin is expressed at similar level. C, Magnetic resonance imaging experiments were performed using a 7 Tesla Pharmascan magnet (Bruker) on anesthetized mice (4 WT and KO) with high resolution T2 images (rapid acquisition with relaxation enhancement: repetition time $=4 \mathrm{~s}$, echo time $=62 \mathrm{~ms}$, matrix $=$ $384 \times 384$, slice thickness $=0.5 \mathrm{~mm}$, resolution $=50 \times 50 \mu \mathrm{m}$, and acquisition time $=38 \mathrm{~min}$ ). $D$, Regions of interest were manually outlined in a blinded fashion using ImageJ. The volume of the third ventricle was significantly higher. $\boldsymbol{E}$, Representative images of $1 \mathrm{DIV}$ cortical explants from WT and $\mathrm{KO}$ embryos, grown in plasma matrix (scale bar: $200 \mu \mathrm{m}$ ). Quantification of maximal axonal length of the different genotypes at 1 DIV (means \pm SEM) (WT, $n=120 ; \mathrm{KO}, n=158$ ) (Student's $t$ test $p=0.831$ ). $\boldsymbol{F}$, Hippocampal neurons from WT and K0 embryos were grown with or without astrocyte-conditioned media for 2 DIV and stained for MAP2 and Tau. Scale bar, $20 \mu \mathrm{m}$. Dendritic and axonal length of WT and KO are not statistically different (Mann-Whitney test: without astrocytes, axons $p=0.46$ and dendrites $p=0.15$; with conditioned media, axons $p=$ 0.16 and dendrites $p=0.28$ ).

\section{Discussion}

Against all predictions based on data in cell lines and cultured primary neurons, here we found that elimination of TIVAMP does not lead to any major developmental defects but show decreased body temperature, increased blood glycerol and increased basal anxiety.

We demonstrated that exon 3 elimination generates a null allele and a complete lack of TI-VAMP expression. The lack of major global developmental defect is surprising considering the role of TI-VAMP in neurite growth, and several secretory pathways in cultured cells (Chaineau et al., 2009) and of TI-VAMP ortholog mutants in plants which show growth defects (Kwon et al., 2008). In absence of TI-VAMP, we found a decrease of brain weight, suggesting the need for a detailed analysis of neuronal architecture. We did not find any defect in growth of TI-VAMP KO cultured neurons in presence or absence of feeding astrocytes. Using hippocampal neurons from a different TI-VAMP KO mouse, Sato et al. (2011) reported a modest effect on axonal growth of neurons grown for 3 DIV. Therefore, we are led to the idea that one or several other v-SNAREs or other mechanisms compensate for TI-VAMP functions. For instance, $\mathrm{KO}$ of endosomal Vtilb generated viable and fertile mice but the invalidation of both Vtila and Vtilb impaired neuronal development and induce perinatal lethality (Kunwar et al., 2011). VAMP4, previously shown to be involved in neurite growth (Racchetti et al., 2010), is an obvious candidate to compensate for the loss of TI-VAMP at least for some of its functions during development. Previous experiments in cultured neurons used silencing approaches and expression of the Longin as dominant-negative fragment to conclude that TI-VAMP was involved in neurite growth. Based on the present data and Sato et al. (2011), it is likely that the abovementioned compensatory mechanisms occur preferentially when TI-VAMP is deleted early in development. We conclude that TI-VAMP functions during development in vivo are either dispensable or compensated for by other SNAREs allowing the KO mice to fulfill major developmental processes.

The main defects identified in our TI-VAMP KO mice are decreased body temperature, increased third ventricle volume, blood glycerol and anxiety. At this point, we cannot relate these phenotypic traits in an obvious manner. Body temperature is regulated by stress and anxiety (Hodges et al., 2008) and increased blood glycerol could be a consequence of compensating the lower temperature through metabolic regulation. Interestingly enough, a polymorphism in Vamp7 was also associated with bipolar affective disorder which is characterized by increased anxiety (Saito et al., 2000; Müller et al., 2002). TI-VAMP is expressed in epithelial cells (Chaineau et al., 2009) thus third ventricle volume defect could be linked to non-neuronal dysfunction. TI-VAMP is the only synaptic v-SNARE cargo of AP-3 (Martinez-Arca et al., 2003). Based on experiments in mocha mice in which TI-VAMP targeting to CA3 
mossy fiber nerve terminals is lost (Scheuber et al., 2006), we proposed that TI-VAMP could be involved in constitutive neurotransmitter release at mossy fiber nerve terminals. The Longin domain of TI-VAMP was recently further proposed to regulate spontaneous release in cultured hippocampal neurons (Hua et al., 2011). Thus, despite the fact that TI-VAMP KO does not show the main neurological deficits of mocha mice (Kantheti et al., 1998), it will now be of high interest to characterize neurotransmission in TI-VAMP KO mice to reveal potential impairment of signaling at a subset of synapses related to unconditioned anxiety and/or thermoregulation and assess its potential role in bipolar affective disorder. TI-VAMP is expressed in neurons and also non-neuronal cells (Chaineau et al., 2009). Therefore, it will be important to characterize conditional mutant specifically lacking expression in neurons or glial cells to further decipher the function of TI-VAMP in both cell types in vivo.

\section{References}

Alberts P, Rudge R, Hinners I, Muzerelle A, Martinez-Arca S, Irinopoulou T, Marthiens V, Tooze S, Rathjen F, Gaspar P, Galli T (2003) Cross talk between tetanus neurotoxin-insensitive vesicle-associated membrane proteinmediated transport and L1-mediated adhesion. Mol Biol Cell 14:4207-4220.

Andrieu D, Meziane H, Marly F, Angelats C, Fernandez PA, Muscatelli F (2006) Sensory defects in Necdin deficient mice result from a loss of sensory neurons correlated within an increase of developmental programmed cell death. BMC Dev Biol 6:56.

Belzung C, Griebel G (2001) Measuring normal and pathological anxietylike behaviour in mice: a review. Behav Brain Res 125:141-149.

Burgo A, Sotirakis E, Simmler MC, Verraes A, Chamot C, Simpson JC, Lanzetti L, Proux-Gillardeaux V, Galli T (2009) Role of Varp, a Rab21 exchange factor and TI-VAMP/VAMP7 partner, in neurite growth. EMBO Rep 10:1117-1124.

Chaineau M, Danglot L, Proux-Gillardeaux V, Galli T (2008) Role of HRB in clathrin-dependent endocytosis. J Biol Chem 283:34365-34373.

Chaineau M, Danglot L, Galli T (2009) Multiple roles of the vesicularSNARE TI-VAMP in post-Golgi and endosomal trafficking. FEBS Lett 583:3817-3826.

Champy MF, Selloum M, Piard L, Zeitler V, Caradec C, Chambon P, Auwerx $\mathrm{J}$ (2004) Mouse functional genomics requires standardization of mouse handling and housing conditions. Mamm Genome 15:768-783.

Danglot L, Triller A, Bessis A (2003) Association of gephyrin with synaptic and extrasynaptic GABAA receptors varies during development in cultured hippocampal neurons. Mol Cell Neurosci 23:264-278.

Danglot L, Chaineau M, Dahan M, Gendron MC, Boggetto N, Perez F, Galli T (2010) Role of TI-VAMP and CD82 in EGFR cell-surface dynamics and signaling. J Cell Sci 123:723-735.

Dawson PA, Steane SE, Markovich D (2005) Impaired memory and olfactory performance in NaSi-1 sulphate transporter deficient mice. Behav Brain Res 159:15-20.

D’Esposito M, Ciccodicola A, Gianfrancesco F, Esposito T, Flagiello L, Mazzarella R, Schlessinger D, D'Urso M (1996) A synaptobrevin-like gene in the Xq28 pseudoautosomal region undergoes X inactivation. Nat Genet 13:227-229.

Gakuba C, Gauberti M, Mazighi M, Defer G, Hanouz JL, Vivien D (2011) Preclinical evidence toward the use of ketamine for recombinant tissuetype plasminogen activator-mediated thrombolysis under anesthesia or sedation. Stroke 42:2947-2949.

Galli T, Zahraoui A, Vaidyanathan VV, Raposo G, Tian JM, Karin M, Niemann H, Louvard D (1998) A novel tetanus neurotoxin-insensitive vesicle-associated membrane protein in SNARE complexes of the apical plasma membrane of epithelial cells. Mol Biol Cell 9:1437-1448.

Gupton SL, Gertler FB (2010) Integrin signaling switches the cytoskeletal and exocytic machinery that drives neuritogenesis. Dev Cell 18:725-736.

Hodges MR, Tattersall GJ, Harris MB, McEvoy SD, Richerson DN, Deneris ES, Johnson RL, Chen ZF, Richerson GB (2008) Defects in breathing and thermoregulation in mice with near-complete absence of central serotonin neurons. J Neurosci 28:2495-2505.

Hua Z, Leal-Ortiz S, Foss SM, Waites CL, Garner CC, Voglmaier SM, Edwards RH (2011) v-SNARE composition distinguishes synaptic vesicle pools. Neuron 71:474-487.

Kantheti P, Qiao X, Diaz ME, Peden AA, Meyer GE, Carskadon SL, Kapfhamer D,
Sufalko D, Robinson MS, Noebels JL, Burmeister M (1998) Mutation in AP-3 delta in the mocha mouse links endosomal transport to storage deficiency in platelets, melanosomes, and synaptic vesicles. Neuron 21:111-122.

Koutnikova H, Laakso M, Lu L, Combe R, Paananen J, Kuulasmaa T, Kuusisto J, Häring HU, Hansen T, Pedersen O, Smith U, Hanefeld M, Williams RW, Auwerx J (2009) Identification of the UBP1 locus as a critical blood pressure determinant using a combination of mouse and human genetics. PLoS Genet 5:e1000591.

Kunwar AJ, Rickmann M, Backofen B, Browski SM, Rosenbusch J, Schöning S, Fleischmann T, Krieglstein K, Fischer von Mollard G (2011) Lack of the endosomal SNAREs vtila and vtilb led to significant impairments in neuronal development. Proc Natl Acad Sci U S A 108:2575-2580.

Kwon C, Neu C, Pajonk S, Yun HS, Lipka U, Humphry M, Bau S, Straus M, Kwaaitaal M, Rampelt H, El Kasmi F, Jürgens G, Parker J, Panstruga R, Lipka V, Schulze-Lefert P (2008) Co-option of a default secretory pathway for plant immune responses. Nature 451:835-840.

Lamprianou S, Vacaresse N, Suzuki Y, Meziane H, Buxbaum JD, Schlessinger J, Harroch S (2006) Receptor protein tyrosine phosphatase gamma is a marker for pyramidal cells and sensory neurons in the nervous system and is not necessary for normal development. Mol Cell Biol 26:5106-5119.

Mandillo S, Tucci V, Hölter SM, Meziane H, Banchaabouchi MA, Kallnik M, Lad HV, Nolan PM, Ouagazzal AM, Coghill EL, Gale K, Golini E, Jacquot S, Krezel W, Parker A, Riet F, Schneider I, Marazziti D, Auwerx J, Brown SD, et al. (2008) Reliability, robustness, and reproducibility in mouse behavioral phenotyping: a cross-laboratory study. Physiol Genomics 34:243-255.

Martinez-Arca S, Alberts P, Zahraoui A, Louvard D, Galli T (2000) Role of tetanus neurotoxin insensitive vesicle-associated membrane protein (TI-VAMP) in vesicular transport mediating neurite outgrowth. J Cell Biol 149:889-900.

Martinez-Arca S, Coco S, Mainguy G, Schenk U, Alberts P, Bouillé P, Mezzina M, Prochiantz A, Matteoli M, Louvard D, Galli T (2001) A common exocytotic mechanism mediates axonal and dendritic outgrowth. J Neurosci 21:3830-3838.

Martinez-Arca S, Rudge R, Vacca M, Raposo G, Camonis J, Proux-Gillardeaux V, Daviet L, Formstecher E, Hamburger A, Filippini F, D’Esposito M, Galli T (2003) A dual mechanism controlling the localization and function of exocytic v-SNAREs. Proc Natl Acad Sci U S A 100:9011-9016.

Masse F, Petit-Demouliere B, Dubois I, Hascoet M, Bourin M (2008) Anxiolytic-like effects of DOI microinjections into the hippocampus (but not the amygdala nor the PAG) in the mice four plates test. Behav Brain Res 188:291-297.

Meziane H, Ouagazzal AM, Aubert L, Wietrzych M, Krezel W (2007) Estrous cycle effects on behavior of C57BL/6J and BALB/cByJ female mice: implications for phenotyping strategies. Genes Brain Behav 6:192-200.

Müller DJ, Schulze TG, Jahnes E, Cichon S, Krauss H, Kesper K, Held T, Maier W, Propping P, Nöthen MM, Rietschel M (2002) Association between a polymorphism in the pseudoautosomal X-linked gene SYBL1 and bipolar affective disorder. Am J Med Genet 114:74-78.

Racchetti G, Lorusso A, Schulte C, Gavello D, Carabelli V, D'Alessandro R, Meldolesi J (2010) Rapid neurite outgrowth in neurosecretory cells and neurons is sustained by the exocytosis of a cytoplasmic organelle, the enlargeosome. J Cell Sci 123:165-170.

Saito T, Parsia S, Papolos DF, Lachman HM (2000) Analysis of the pseudoautosomal X-linked gene SYBLlin bipolar affective disorder: description of a new candidate allele for psychiatric disorders. Am J Med Genet 96:317-323.

Sato M, Yoshimura S, Hirai R, Goto A, Kunii M, Atik N, Sato T, Sato K, Harada R, Shimada J, Hatabu T, Yorifuji H, Harada A (2011) The role of VAMP7/TI-VAMP in cell polarity and lysosomal exocytosis in vivo. Traffic 12:1383-1393.

Scheuber A, Rudge R, Danglot L, Raposo G, Binz T, Poncer JC, Galli T (2006) Loss of AP-3 function affects spontaneous and evoked release at hippocampal mossy fiber synapses. Proc Natl Acad Sci U S A 103:16562-16567.

Schwenk RW, Dirkx E, Coumans WA, Bonen A, Klip A, Glatz JF, Luiken JJ (2010) Requirement for distinct vesicle-associated membrane proteins in insulin- and AMP-activated protein kinase (AMPK)-induced translocation of GLUT4 and CD36 in cultured cardiomyocytes. Diabetologia 53:2209-2219.

Vacca M, Albania L, Della Ragione F, Carpi A, Rossi V, Strazzullo M, De Franceschi N, Rossetto O, Filippini F, D'Esposito M (2011) Alternative splicing of the human gene SYBL1 modulates protein domain architecture of Longin VAMP7/TI-VAMP, showing both non-SNARE and synaptobrevin-like isoforms. BMC Mol Biol 12:26.

Weber T, Zemelman BV, McNew JA, Westermann B, Gmachl M, Parlati F, Söllner TH, Rothman JE (1998) SNAREpins: minimal machinery for membrane fusion. Cell 92:759-772. 